\title{
Stress induced magnetic anisotropy and giant magnetoimpedance in Fe-rich glass-coated magnetic microwires
}

\author{
V. Zhukova \\ "TAMag Iberica” S.L., Avda de los Remedios 41-3", Colmenar Viejo, Madrid, Spain \\ V. S. Larin \\ "TAMag Iberica" S.L., Avda de los Remedios 41-3", Colmenar Viejo, Madrid, Spain, \\ and "AmoTec" S. R. L., 5, Miorita str., 2028 Kishinev, Republic of Moldova
}

\author{
A. Zhukova) \\ Instituto de Ciencia de Materiales, CSIC. 28049 Cantoblanco, Madrid, Spain, \\ and "TAMag Iberica" S. L., Avda de los Remedios 41-3a, Colmenar Viejo, Madrid, Spain
}

(Received 24 January 2003; accepted 28 April 2003)

\begin{abstract}
The effect of conventional (CA) and stress annealing (SA) on magnetic properties of $\mathrm{Fe}_{74} \mathrm{~B}_{13} \mathrm{Si}_{11} \mathrm{C}_{2}$ glass-coated microwires has been studied. CA treatment does not significantly change the character of the hysteresis loop. Under certain annealing conditions (annealing temperature, $T_{\text {ann }}>300{ }^{\circ} \mathrm{C}$, applied stress, $\sigma>700 \mathrm{MPa}$ ) rectangular hysteresis loop transforms into the inclined with magnetic anisotropy field above $1000 \mathrm{~A} / \mathrm{m}$. Such phenomenology has been related to the induction of transverse magnetic anisotropy by SA treatment. Under tensile stress the SA annealed microwire recovers rectangular hysteresis loop. Samples subjected to stress annealing show noticeable magnetoimpedance and stress impedance effects in spite of their large magnetostriction. (C) 2003 American Institute of Physics. [DOI: 10.1063/1.1585113]
\end{abstract}

\section{INTRODUCTION}

The ferromagnetic wires obtained by the in-rotatingwater quenching technique (diameter of around $120 \mu \mathrm{m}$ ) attract permanent attention owing to their peculiar magnetization properties, such as so-called magnetic bistability in magnetostrictive compositions ${ }^{1}$ or the giant magnetoimpedance (GMI) effect in nonmagnetostrictive compositions. $^{2}$

On the other hand, Taylor-Ulitovsky technology $y^{3,4}$ allows one to produce much tinier metallic microwires (the metallic nucleus diameter is between 1 and $20 \mu \mathrm{m}$ ) coated by insulating glass (the glass coating thickness is between 1 and $10 \mu \mathrm{m}$ ). Initially most attention was paid to studies of mechanical and electrical properties of such microwire, ${ }^{5}$ while less attention was paid over these years to their magnetic properties. ${ }^{6,7}$ The peculiar magnetic properties found in these tiny glass-coated microwires (their magnetic softness, GMI effect and magnetic bistability) renewed interest in such microwires. ${ }^{8,9}$

The hysteretic magnetic properties of glass-coated microwires are determined by the value and sign of the magnetostriction constant, $\lambda_{s}$. Thus, Fe-rich compositions with $\lambda_{s}>0$ exhibit rectangular hysteresis loop, ${ }^{8,9}$ while negative magnetostriction alloy microwires have inclined magnetization curves with low coercivity, $H_{c} \cdot{ }^{8,10}$ Certain efforts have been performed in order to achieve an enhanced magnetic softness in glass coated microwires. ${ }^{11}$ It has been found that the compositional range of alloys exhibiting soft magnetic properties is quite narrow. ${ }^{11}$ On the other hand, the adequate choosing of the alloy composition, geometric parameters and

a) Author to whom correspondence should be addressed; electronic mail: arcadyzh@icmm.csic.es conditions of annealing can significantly enhance soft magnetic properties and GMI effect (up to 600\%) and also extend the compositional range of alloys with enhanced magnetic softness. $^{11-14}$

In fact, Fe-rich amorphous microwires with rectangular hysteresis loop exhibit rather poor initial magnetic permeability and do not show GMI effect due to the strong longitudinal magnetic anisotropy. Consequently the challenge of this article is to present novel results on tailoring of magnetic properties of Fe-rich microwires by means of induction of transverse magnetic anisotropy.

\section{EXPERIMENTAL TECHNIQUE AND RESULTS}

Amorphous $\mathrm{Fe}_{74} \mathrm{~B}_{13} \mathrm{Si}_{11} \mathrm{C}_{2}$ microwires with metallic nucleus diameter, $d=15 \mu \mathrm{m}$ and total diameter, $D=22 \mu \mathrm{m}$ were obtained by the Taylor-Ulitovsky technique. ${ }^{3-7,15}$

The longitudinal $M-H$ hysteresis loop of the samples was obtained at room temperature by means of an induction method at $50 \mathrm{~Hz}$. The magnetic field, $H$, was applied by a Helmholtz coil system producing a maximum field of 5 $\mathrm{kA} / \mathrm{m}$. The experimental setup allowed us also to apply tensile strain on the samples, such as described elsewhere. ${ }^{11}$

Conventional furnace annealing without applied stress (CA) and under applied tensile stress (SA) at temperatures, $T_{\text {ann }}$, below crystallization temperature has been performed. It is worth mentioning that for the SA annealing the sample has been first loaded by the stress and then placed in the furnace.

The applied tensile stress within the metallic nucleus and glass shell has been calculated as ${ }^{16}$ 

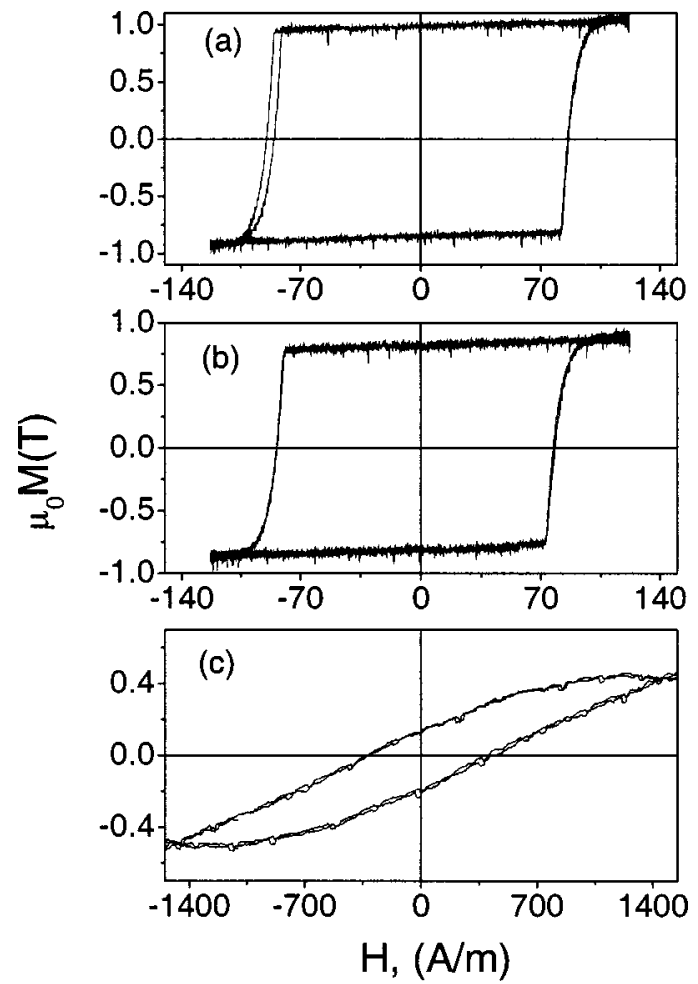

FIG. 1. Hysteresis loop of $\mathrm{Fe}_{74} \mathrm{~B}_{13} \mathrm{Si}_{11} \mathrm{C}_{2}$ glass-coated microwire in asprepared state (a), after $\mathrm{CA}(\mathrm{b})$ and after $\mathrm{SA}(\mathrm{c})$.

$$
\begin{gathered}
\sigma_{m}=\frac{k P}{k S_{m}+S_{\mathrm{gl}}}, \\
\sigma_{g l}=\frac{P}{k S_{m}+S_{\mathrm{gl}}},
\end{gathered}
$$

where $k=E_{2} / E_{1}, E_{i}$ are the Young modulus of metal $\left(E_{2}\right)$ and glass $\left(E_{1}\right)$ at room temperature and $P$ is the applied mechanical load.

The impedance, $Z$, of the microwire was evaluated by means of the four-point technique. The magneto-impedance ratio, $\Delta Z / Z_{H}$, has been defined as

$$
\Delta Z / Z_{H}=\left[Z(H)-Z\left(H_{\max }\right)\right] / Z\left(H_{\max }\right) .
$$

A maximum de longitudinal magnetic field, $H_{\max }$, up to $2500 \mathrm{~A} / \mathrm{m}$ was supplied by a long solenoid.

The stress-impedance ratio, $\Delta Z / Z_{\sigma}$, has been defined as

$$
\Delta Z / Z_{\sigma}=\left[Z(\sigma)-Z\left(\sigma_{\max }\right)\right] / Z\left(\sigma_{\max }\right) .
$$

A maximum tensile stress, $\sigma_{\max }$, up to $370 \mathrm{MPa}$ was supplied by an attached load.

As expected, ${ }^{17}$ the hysteresis loop of as-prepared microwire exhibits spontaneous magnetic bistability [see Fig. 1(a)]. Conventional annealing performed at $T_{\text {ann }}=400{ }^{\circ} \mathrm{C}$ does not significantly affect the magnetic properties of studied sample [see Fig. 1(b)]: some decrease of $H_{c}$ has been observed while the squared character of the hysteresis loop remains unchanged. On the other hand, SA performed at the same annealing conditions $\left(T_{\text {ann }}=400{ }^{\circ} \mathrm{C}\right)$ but under applied stress, $\sigma=458 \mathrm{MPa}$, results in drastic changes of the hysteresis loop [see Fig. 1(c)]: hysteresis loop becomes inclined with a magnetic anisotropy field about $1000 \mathrm{~A} / \mathrm{m}$.

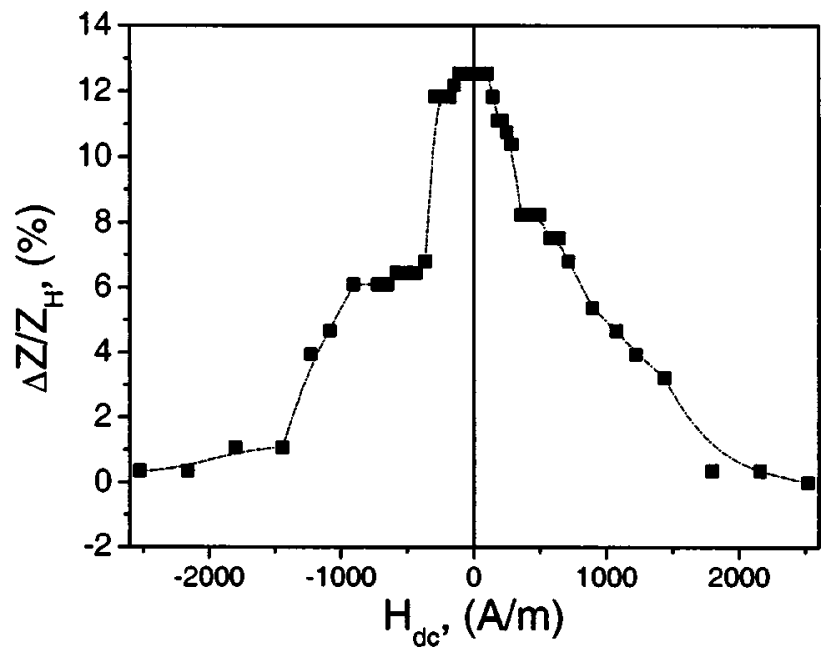

FIG. 2. GMI effect of annealed under stress (468 MPa) at $400{ }^{\circ} \mathrm{C}$ for $t$ $=170 \mathrm{~s} \mathrm{Fe}_{74} \mathrm{~B}_{13} \mathrm{Si}_{11} \mathrm{C}_{2}$ glass-coated microwire measured at $10 \mathrm{MHz}$ for the driving current amplitude of $2 \mathrm{~mA}$.

A transverse magnetic anisotropy deduced from the hysteresis loop shape of the sample subjected to SA [see Fig. $1(\mathrm{c})]$ allows us to predict the existence of the magnetoimpedance effect in such samples. Indeed, if as-prepared samples do not presented any GMI effect, the samples subjected to SA exhibit significant (around 12\%) magnetoimpedance (see Fig. 2), having a shape of monotonic decay from $H=0$ (typical for $\lambda_{s}>0$ ).

Another important result is that the application of tensile stresses to the samples subjected to the SA treatment results in the recovery of the rectangular hysteresis loop typical for the as-prepared state (see Fig. 3).

Consequently, the disappearance of the inclined hysteresis loop under applied stress allows as to predict stress impedance effect, SI, in the sample subjected to SA treatment. Indeed considerable SI effect (about 13\%) has been observed under application of tensile stress (see Fig. 4).

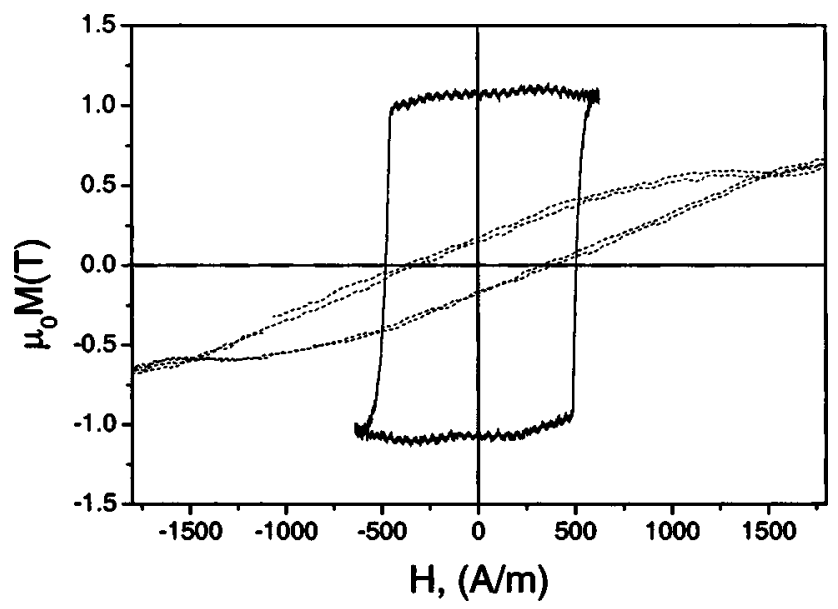

FIG. 3. Hysteresis loop of $\mathrm{SA} \mathrm{Fe}_{74} \mathrm{~B}_{13} \mathrm{Si}_{11} \mathrm{C}_{2}$ glass-coated microwire measured under applied stress of $500 \mathrm{MPA}$. Hysteresis loop of $\mathrm{Fe}_{74} \mathrm{~B}_{13} \mathrm{Si}_{11} \mathrm{C}_{2}$ subjected to SA without stress has been shown for the comparison by dashed line. 


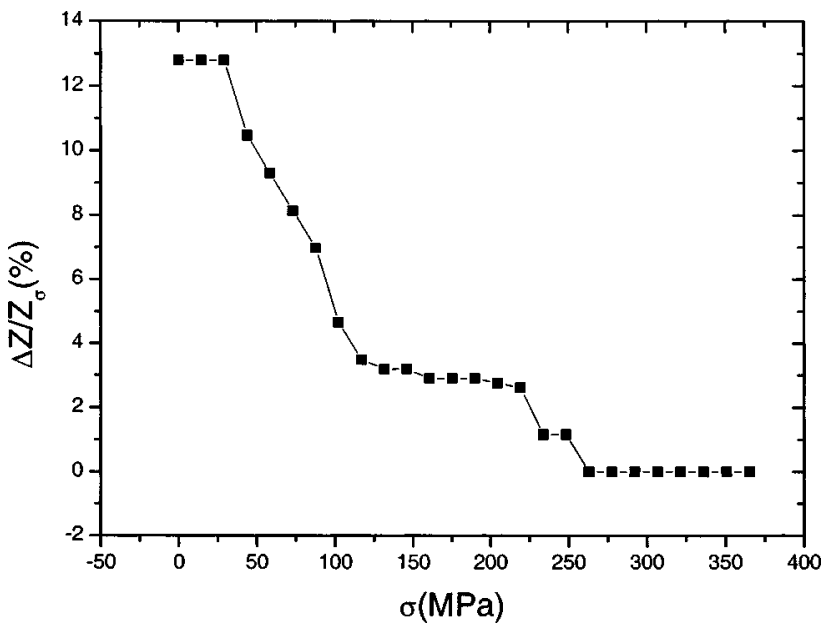

FIG. 4. Stress impedance effect of annealing under stress (468 MPa) at 400 ${ }^{\circ} \mathrm{C}$ for $t=170 \mathrm{~s} \mathrm{Fe}_{74} \mathrm{~B}_{13} \mathrm{Si}_{11} \mathrm{C}_{2}$ glass-coated microwire measured at $10 \mathrm{MHz}$ for the driving current amplitude of $2 \mathrm{~mA}$.

\section{DISCUSSION}

The origin of this creep annealing induced anisotropy can be attributed to both: (i) redistribution of the residual stresses during the stress annealing or (ii) induced magnetic anisotropy related to the atomic ordering.

The peculiarity of studied materials is that they have additional source of the internal stresses related with the difference in the thermal expansion coefficients of metallic nucleus and glass coating. ${ }^{4,8-11,18}$ It should be assumed that after the thermal treatment the longitudinal stresses in stress annealed samples become smaller than radial and tangential stresses, i.e., that the stress annealing results in redistribution of the internal stresses and/or local microstructure of the sample in order to minimize the magnetoelastic energy in the stressed state. As a result the easy magnetization axis is aligned along the transverse direction. In this way, the removal of the mechanical load after stress annealing affects mostly the longitudinal stresses and results in drastic decrease of the longitudinal stress component and even in the appearance of the compressive longitudinal stresses (socalled "back stresses").

Observed change of the hysteresis loop of the sample subjected to SA treatment when measured under applied tensile stress (Fig. 3) confirms such assumption. This effect should be attributed to the increasing of the longitudinal stress component under application of the tensile stress and consequently to the alignment of the easy magnetization axis along the highest stress component due to the positive magnetostriction constant of the studied sample. There is a competition between the magnetoelastic anisotropy induced by the applied stress (with a longitudinal easy axis) and the transverse anisotropy induced by the stress annealing. Upon overcoming the transverse anisotropy related to creep induced anisotropy the longitudinal axis becomes easy magnetization axis again and magnetic bistability recovers.

Theoretical and experimental studies showed that the longitudinal stress components are generally larger than tangential or radial. ${ }^{10,12,18,19}$ The magnetic domain structure of the glass-coated microwire depends on the magnetoelastic energy (roughly proportional to the $\lambda_{s} \sigma_{i}$, where $\sigma_{i}$ is the internal stress), magnetostatic energy and exchange energy.

In the case of the rectangular hysteresis loop the coercivity should be proportional to the energy required to form the domain wall $\gamma$ involved in the bistable magnetization process. The domain wall energy related to the magnetoelastic anisotropy and then to the applied tensile stress is given by $^{20}$

$$
H_{c} \propto \gamma \propto \frac{\left[A(3 / 2) \lambda_{s}\left(\sigma_{a}+\sigma_{r}\right)\right]^{1 / 2}}{\cos \alpha},
$$

where $\alpha$ is the angle between magnetization and longitudinal direction, $A$ is the exchange energy constant and $\sigma_{r}$ is the residual tensile stress. Consequently, $H_{c}$ must be proportional to $\sigma_{a}^{1 / 2}$ for $\sigma_{a}$ larger than $\sigma_{r}$ and $\cos \alpha \approx 1$. On the other hand, internal stress relaxation (decreasing of $\sigma_{r}$ ) due to conventional heat treatment originates some decrease of the coercivity.

In the most simple approximation ${ }^{4,7,19}$ the strength of the internal stresses (longitudinal, $\sigma_{z}$, radial, $\sigma_{r}$, and tangential, $\sigma_{\phi}$, components) depends on the volume of the glass coating and metallic nucleus as follows:

$$
\begin{aligned}
& \sigma_{z}=\sigma_{0} \frac{k x}{k x+1} \cdot \frac{(k+1) x+1}{(k / 3+1) x+4 / 3}, \\
& \sigma_{r}=\sigma_{\phi}=\sigma_{0} \cdot \frac{k x}{(k / 3+1) x+4 / 3},
\end{aligned}
$$

where $\quad x=D^{2} /\left(d^{2}-1\right), \quad \sigma_{0}=E_{1}\left(a_{1}-a_{2}\right)\left(T^{*}-T\right)$, $T^{*}$-solidification temperature of the composite wire (i.e., when both metallic nucleus and glass coating solidify), $T$-room temperature, $k=E_{2} / E_{1}=0.3-0.6, \quad a_{1}$ and $a_{2}$-thermal expansion coefficients of the glass and the metal, respectively.

Similar to as was described for creep anisotropies related with short term atomic ordering in the other kind of amorphous materials with positive $\lambda_{S},{ }^{21,22}$ transverse magnetic anisotropy has been induced in the present case. Observed effect is clearly related with a stress induced magnetic anisotropy. In this way, "plastic" and "anelastic" ordering ${ }^{22}$ can be considered for the explanation of observed phenomenology. To justify the observed phenomenology, it seems reasonable to assume that creep induced anisotropy overcomes the intrinsic magnetoelastic anisotropy. The alternative origin of the induced magnetic anisotropy related to the pair ordering mechanism is assumed to be less probable since observed phenomenon is clearly related with the stress applied during the heat treatment and because only one transition metal is presented in the alloy composition.

Finally, observed GMI and stress-impedance effects should be attributed to the induction of transverse magnetic anisotropy.

\section{CONCLUSIONS}

Concluding, stress annealing of $\mathrm{Fe}_{74} \mathrm{~B}_{13} \mathrm{Si}_{11} \mathrm{C}_{2}$ glasscoated microwire results in transformation of the initially rectangular hysteresis loop to the inclined hysteresis loop. GMI effect and stress-impedance effect are observed in such stress annealed samples. Application of tensile stresses in the 
samples subjected to the SA treatment results in the appearance of the rectangular hysteresis loop typical for the asprepared state.

The appearance of such hysteresis loop is explained by the creep induced magnetic anisotropy and/or redistribution of the internal stresses arising from the difference of the thermal expansion coefficients of the metal and glass during SA during such stress annealing.

\section{ACKNOWLEDGMENT}

The authors acknowledge useful discussions with Professor M. Vázquez.

${ }^{1}$ K. Mohri, F. B. Humphrey, K. Kawashima, K. Kimura, and M. Muzutani, IEEE Trans. Magn. Mag-26, 1789 (1990).

${ }^{2}$ R. L. V. Panina and K. Mohri, Appl. Phys. Lett. 65, 1189 (1994).

${ }^{3}$ A. V. Ulitovski, USSR Patent No. 128427, 3.9.1950.

${ }^{4}$ E. Ia. Badinter, N. R. Berman, I. F. Drabenco, V. I. Zaborovski, Z. I. Zelikovski, and V. G. Cheban, Cast Microwire and Its properties (Shtinitsa, Kishinev, 1973), pp. 6-196.

${ }^{5}$ T. Goto, Trans. Jpn. Inst. Met. 21, 219 (1980).

${ }^{6}$ L. Kraus, J. Schneider, and H. Wiesner, Czech. J. Phys., Sect. B 26, 601 (1976).

${ }^{7}$ S. A. Baranov, V. N. Berzhanski, S. K. Zotov, V. L. Kokoz, V. S. Larin, and A. V. Torcunov, Phys. Met. Metallogr. 67, 73 (1989).
${ }^{8}$ M. Vázquez, and A. Zhukov, J. Magn. Magn. Mater. 160, 223 (1996).

${ }^{9}$ H. Chiriac and T. A. Ovari, in Progress in Material Science (Elsevier, London, 1997), Vol. 40, p. 333.

${ }^{10}$ J. Velázquez, M. Vázquez and A. Zhukov, J. Mater. Res. 11, 2499 (1996).

${ }^{11}$ A. F. Cobeño, A. Zhukov, A. R. de Arellano-Lopez, F. Elías. J. M. Blanco, V. Larin, and J. González, J. Mater. Res. 14, 3775 (1999).

${ }^{12}$ A. Zhukov, J. Magn. Magn. Mater. 242-245, 216 (2002).

${ }^{13}$ K. R. Pirota, L. Kraus, H. Chiriac, and M. Knobel, J. Magn. Magn. Mater. 221, L243 (2000).

${ }^{14}$ A. Zhukov, J. Gonzalez, J. M. Blanco, M. J. Prieto, E. Pina, and M. Vazquez, J. Appl. Phys. 87, 1402 (2000).

${ }^{15}$ G. F. Taylor, Phys. Rev. 24, 655 (1924).

${ }^{16}$ E. Ia. Badinter and E. M. Lysko, Microwire and resistivity devices (Shtinitsa, Kishinev, 1962), pp. 52-62.

${ }^{17}$ A. P. Zhukov, M. Vázquez, J. Velázquez, H. Chiriac, and V. Larin, J. Magn. Magn. Mater. 151, 132 (1995)

${ }^{18}$ A. S. Antonov, V. T. Borisov, O. V. Borisov, A. F. Prokoshin, and N. A. Usov, J. Phys. D 33, 1161 (2000).

${ }^{19}$ A. Zhukov, J. Gonzalez, A. Torcunov, E. Pina, M. J Prieto, A. F. Cobeño, J. M. Blanco, V. Larin, and S. Baranov, J. Magn. Magn. Mater. 203, 238 (1999).

${ }^{20}$ J. Gonzalez, N. Murillo, V. Larin, J. M. Barandiaran, M. Vazquez, and A. Hernando, IEEE Trans. Magn. 33, 2362 (1997).

${ }^{21}$ A. Hernando, M. Vázquez, and J. M. Barandiaran, J. Phys. E 21, 1129 (1988).

${ }^{22}$ M. Vasquez, E. Ascasibar, A. Hernando, and O. V. Nelson, J. Magn. Magn. Mater. 66, 37 (1987). 